\title{
Mehr Frauen in deutsche Aufsichtsräte!
}

\section{Statement anlässlich des djb-Frauen-Dinners am 18. Oktober 2007, Berlin}

Meine Damen, meine Herren, vielen Dank, dass Sie unserer Veranstaltung und unserem Anliegen heute Abend Ihre Aufmerksamkeit widmen.

Mehr Frauen in deutsche Aufsichtsräte! Sie werden sich vielleicht fragen, was nun ausgerechnet der Deutsche Juristinnenbund mit einem - auf den ersten Blick - rein wirtschaftlichen Thema zu schaffen hat. Die Antwort lautet schlicht: Es ist einfach kein rein wirtschaftliches Thema.

Ganz allgemein sind Frauen in Führungspositionen heute zwar keine Exoten mehr, dennoch sind wir in Deutschland aus verschiedenen Gründen weit davon entfernt, ebenso viele Frauen wie Männer in mittleren und hohen Führungsebenen anzutreffen. Ganz besonders prekär ist die Situation bei hochqualifizierten Führungspositionen. Frauen in Vorständen deutscher Unternehmen, so zeigen es jüngste Untersuchungen, sind selten. Es gibt zum Beispiel nur fünf Frauen in den Vorständen der 30 DAX- und 50 MDAX-Unternehmen. Immerhin, werden Sie jetzt vielleicht sagen. Bei insgesamt 419 Vorstandsmitgliedern (194 in den DAXund 225 in den MDAX-Unternehmen) bewirkt die Zahl von nur fünf Frauen aber nur eine Quote von weniger als 1,2 Prozent.

Der djb sagt ganz klar: Das steht einer führenden Wirtschaftsnation nicht gut zu Gesicht, das ist absolut unzeitgemäß!

Leider sieht es aber in den deutschen Aufsichtsräten nicht anders aus. Das zeigt eine Untersuchung des European Professional Women's Network aus dem Juni 2006. Diese Untersuchung beschäftigte sich mit dem Anteil von Frauen in Führungsgremien europäischer Unternehmen.

Dieser BoardWomen Monitor 2006 untersuchte die Board-Zusammensetzung der 300 größten europäischen Unternehmen (nach dem FTSEurofirst 300 Index). Das Ergebnis für Deutschland war eher ernüchternd!

Hierzu möchte ich kurz zum Verständnis einfügen, dass als Board-Mitglieder Executive und Non-executive Directors berücksichtigt wurden, also nach deutschem Rechtsverständnis Vorstände und Aufsichtsräte. In dieser Untersuchung werden sowohl Unternehmensführungen mit einheitlichen Leitungsorganen als auch mit dualen Führungssystemen als Board bezeichnet.
Der Board Women Monitor zeigt, dass der Frauenanteil in den obersten Führungsetagen der größten europäischen Unternehmen erheblich divergiert. Die norwegischen Unternehmen hatten einen Frauenanteil von fast 30 Prozent (genau 28,8 Prozent), die portugiesischen Firmen konnten dagegen nicht eine einzige Frau in der Unternehmensspitze vorweisen. Auch die anderen skandinavischen Länder finden sich an der Spitze der Tabelle wieder: Schweden steht mit fast 23 Prozent (genau 22,8 Prozent) nach Norwegen an der zweiten Position, Finnland mit 20 Prozent an dritter und Dänemark mit knapp 18 Prozent (17,9 Prozent) an vierter Stelle.

Deutschland hingegen kommt nur auf 7,2 Prozent. Damit liegen die deutschen Unternehmen in der Mitte des Untersuchungsfeldes. Allerdings haben sie diese Position nur aufgrund der deutschen Mitbestimmungsregelungen inne, denn die meisten Frauen werden von den Arbeitnehmervertretungen in die Aufsichtsräte entsandt.

Berücksichtigt man nur die Seite der Anteilseigner, zeichnet sich ein ganz anderes Bild. Sie haben nämlich nur drei Prozent ihrer Mandate an Frauen vergeben. Damit rutscht Deutschland in den Kreis der europäischen Schlusslichter bei der Besetzung von Board-Positionen mit Frauen. Nur in Portugal und Italien sind noch weniger Frauen in den Spitzen der Unternehmen tätig.

Natürlich stellt sich in diesem Zusammenhang die Frage, warum es so signifikante Unterschiede gibt. Naheliegend wäre zu sagen, norwegische Frauen sind einfach klüger und machtbewusster. Man könnte auch noch über ihre ethnischen Wurzeln aus der Wikingerzeit fabulieren, aber glauben Sie mir, das führt nicht zu wirklichen Erhellungen. Ein Vergleich der Rechtsgrundlagen und des rechtspolitischen Hintergrunds bringt da schon mehr Licht ins Dunkel.

Und jetzt schließt sich für Sie auch der Kreis, warum sich neben dem Verband deutscher Unternehmerinnen (VDU) oder dem Verein Victress Initiative hier in Berlin und anderen auch der Deutsche Juristinnenbund in dieser Frage engagiert. In unserer Kommission „Arbeits-, Gleichstellungsund Wirtschaftsrecht" unter Leitung von Frau Prof. Dr. Sybille Raasch haben wir bereits Anfang des Jahres 2006 eine Untergruppe „Corporate
Jutta Wagner

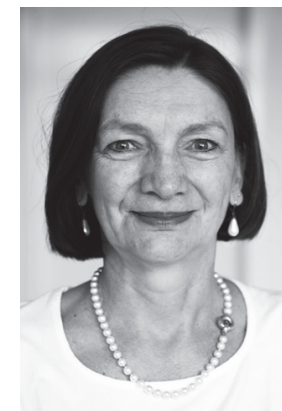

Jutta Wagner

Präsidentin des djb,

Rechtsanwältin und

Notarin, Berlin 
Governance“ gebildet. In dieser Untergruppe haben sich ausgewiesene Expertinnen des Arbeits-, Gleichstellungs- und Wirtschaftsrechts, des Öffentlichen und des Zivilrechts sowie aus dem Bereich Personalmanagement und aus der Wissenschaft mit dem Thema „Frauen in Führungspositionen“ beschäftigt. Die Ergebnisse dieser Arbeit haben zu unserer djb-Initiative „Mehr Frauen in deutsche Aufsichtsräte!“ geführt.

Der Anstoß für unsere Arbeit kam - natürlich aus Norwegen. Norwegen war weltweit das erste Land, das eine gesetzliche Quotenregelung für die Board-Besetzung von Aktiengesellschaften eingeführt hat. Die Regelung trat mit dem Aktiengesetz vom 19. Dezember 2003 in Kraft und sieht eine

\section{Ist der norwegische - oder wenn man die Erfolge in den anderen skandinavischen Ländern betrachtet - der skandinavische Weg der Königsweg?}

Quotierung für Anteilseignervertreter vor. Von diesem Gesetz sind gegenwärtig etwa 500 Unternehmen betroffen. Da norwegische Aktiengesellschaften monistisch organisiert sind, betrifft die Quotenregelung Executive und Non-executive Directors. In deutsche gesellschaftsrechtliche Kategorien übersetzt bedeutet das, dass Personen, die Aufsichtsrats- und Vorstandstätigkeiten wahrnehmen, davon betroffen sind. Da die Mitglieder der Geschäftsführung in Norwegen nur selten einen Sitz im Board des Unternehmens haben, kann man diese Rechtskategorie so interpretieren, dass sie sich insbesondere auf Aufsichtsratsmitglieder bezieht.

Ich möchte Sie jetzt nicht in die Tiefen des norwegischen Aktienrechts entführen, das würde hier heute den Rahmen sprengen. Ich möchte aber erwähnen, dass die norwegische Rechtsregelung sehr strikt ist und in allerletzter Konsequenz sogar die Auflösung einer Gesellschaft vorsieht, die nur in begründeten Ausnahmen durch den norwegischen König verhindert werden kann.

Selbstverständlich sind solche gesetzlichen Regelungen von erheblicher Tragweite für das Wirtschaftsleben. Das hat auch der norwegische Industrieverband erkannt. Nach anfänglichen Widerständen unterstützt der Verband nun seine Mitgliedsunternehmen dabei, die Quote erfüllen zu können. Er entwickelte das Programm „Female Future“, in dem weibliche Führungskräfte für Board-Positionen gezielt ausgebildet und gefördert werden. Der Verband listet in einer eigenen Datenbank die Namen von Frauen auf, die am Female-
Future-Programm teilgenommen haben. Daneben gibt es eine Reihe von Datenbanken, die Unternehmen dabei unterstützen, geeignete Kandidatinnen zu finden.

Sie wundern sich jetzt vielleicht über diese strikten, vielleicht sogar radikal anmutenden norwegischen Regeln. Aber bei genauerer Betrachtung stellt man fest, dass Norwegen seit langem eine Vorreiterrolle bei der aktiven Gleichstellungspolitik einnimmt. Bereits Ende der 1970er Jahre hat Norwegen ein Gesetz zur Gleichstellung der Geschlechter verabschiedet und war damit weltweit das erste Land.

Dass die erste Ministerpräsidentin Gro Harlem Brundtland ihr Kabinett mit über 40 Prozent Ministerinnen besetzte, war also nur folgerichtig, trotzdem sorgte dieser Umstand weltweit für Schlagzeilen.

Ist der norwegische - oder wenn man die Erfolge in den anderen skandinavischen Ländern betrachtet - der skandinavische Weg der Königsweg? Diese Frage kann natürlich nur die deutsche Wirtschaft beantworten. Auch in Norwegen hat man vor der Quotenregelung auf das Prinzip Freiwilligkeit gesetzt. Das fertig ausgearbeitete Gesetz wurde in die Schublade gelegt und der Wirtschaft eine klare Frist gesetzt. Das Ziel war, einen Frauenanteil in den Aufsichtsräten von 40 Prozent zu erreichen. Die Unternehmen ließen die Frist verstreichen. Das Gesetz trat in Kraft.

Auch wenn die Quotenregelung umstritten bleibt: Seit Androhung dieser Regel über ihre Umsetzung bis zum heutigen Zeitpunkt hat sich der Frauenanteil in den norwegischen Boards signifikant erhöht: von sechs Prozent im Jahr 2002 auf 11 Prozent im Jahr 2004 und 25 Prozent im Jahr 2007. Bereits 38 Prozent der norwegischen Aktiengesellschaften erfüllen die 40-Prozent-Klausel. Also doch der norwegische Weg?

In Deutschland sehen die Rechtsgrundlagen ganz anders aus. Das deutsche Aktiengesetz ordnet an, dass Mitglied des Aufsichtsrats nur natürliche und uneingeschränkt geschäftsfähige Personen sein können. Es verlangt weder formale Qualifikationen oder Fähigkeiten, noch stellt es geschlechtsspezifische Forderungen auf.

Der Deutsche Corporate Governance Kodex wird konkreter und präzisiert die geforderten Qualifikationen und Fähigkeiten. Aber auch diese Besetzungsregelung ist geschlechtsneutral formuliert.

Der Deutsche Corporate Governance Kodex wurde übrigens von der gleichnamigen Regierungskommission im Februar 2002 verabschiedet. Seither wurde er immer wieder aktualisiert, nur in 
der geschlechtsspezifischen Frage eben nicht. Leider ist auch die Regierungskommission, was das Geschlecht ihrer Mitglieder betrifft, sehr einseitig besetzt: 13 Mitglieder, keine Frau!

Den bisher einzigen Lichtblick gibt das Mitbestimmungsrecht. Trotz der geschlechtsneutralen Besetzungsregelungen für die Anteilseignervertreter sieht das Gesetz für Arbeitnehmervertreter eine geschlechtsspezifische Regelung vor: Frauen und Männer sollen hier entsprechend ihrem zahlenmäßigen Verhältnis im Unternehmen beteiligt sein. Die Sollvorschrift hat Signalwirkung mit praktischem Erfolg: 80 Prozent der weiblichen Aufsichtsratsmitglieder sind Arbeitnehmervertreterinnen. Übrigens gibt es für die vom Bund zu vergebenden Aufsichtsratsmandate ebenfalls geschlechtsspezifische Besetzungsregelungen.

Auch von dieser gleichstellungspolitischen Maßnahme ist eine gewisse Signalwirkung ausgegangen. Nach Inkrafttreten des Bundesgremienbesetzungsgesetzes ist der Frauenanteil in Gremien mit Bundeseinfluss von 1990 bis 2005 langsam aber stetig von 7,7 Prozent auf 20,5 Prozent gestiegen.

Trotzdem müssen wir feststellen, dass aktive Gleichstellungspolitik in Deutschland auf den öffentlichen Bereich begrenzt ist. Mit Gründung der GRÜNEN im Jahr 1979 begann in Deutschland eine Quotendiskussion. Die GRÜNEN haben entsprechende Regelungen getroffen. 1988 zog die SPD nach und 1996 hat dann auch die CDU nach langer Diskussion positive Maßnahmen zur Erhöhung des Frauenanteils eingeführt. Seit den 1980er Jahren begannen fast alle Bundesländer und 1994 dann endlich auch der Bund Gleichstellungsgesetze für den öffentlichen Dienst einzuführen. Der öffentliche Sektor konnte in Deutschland aber nicht wie in Skandinavien zum Vorreiter für die Privatwirtschaft werden. Im Koalitionsvertrag der Rot/Grünen-Bundesregierung von 1998 wurde ein effektives Gleichstellungsgesetz mit verbindlichen Regelungen für die Privatwirtschaft angekündigt. Trotz vorliegender Gesetzentwürfe legte die Regierung das Vorhaben wieder auf Eis und setzte auf einen Dialog mit der Wirtschaft.

Es gibt mittlerweile eine Reihe von empirischen Untersuchungen, die einen Zusammenhang zwischen dem Erfolg eines Unternehmens und der ausgewogenen Besetzung seiner Boards erkennen lassen. Es stellt sich also auch die Frage, warum gewinnorientierte Unternehmen nicht aus eigenem ökonomischem Interesse den Vorteil ausgewogen besetzter Führungsgremien nutzen. Fest steht nur, dass sie es nicht tun.
Aufgrund dieser Erfahrung und des geringen Anteils von Frauen in Führungspositionen vereinbarte die Bundesregierung dann im Jahr 2001 mit den Spitzenverbänden der deutschen Wirtschaft eine Förderung der Chancengleichheit von Frauen und Männern in der Privatwirtschaft. Die Bundesregierung verpflichtet sich darin, keine Initiative zu ergreifen, die Chancengleichheit von Frauen und Männern auf gesetzlichem Wege zu erreichen, solange die Vereinbarung erfolgreich umgesetzt wird. Die Umsetzung der Vereinbarung wird alle zwei Jahre überprüft.

Und obwohl die Ergebnisse ernüchternd sind und nur geringe Fortschritte bei der Umsetzung zu verzeichnen sind, wurde die Verabschiedung gesetzgeberischer Vorhaben - zunächst - aufgegeben. Es gibt also gegenwärtig in Deutschland keine positiven Maßnahmen zur Erhöhung des Frauenanteils bei Aufsichtsratsmandaten von Anteilseignern in deutschen Privatunternehmen.

Der Deutsche Juristinnenbund positioniert sich hier sehr eindeutig: Es wird Zeit, dass sich das ändert. Wir setzen heute mit unserem „Frauen-Dinner" ein Zeichen. Es gibt sehr viele qualifizierte Frauen in der deutschen Wirtschaft, die sehr wohl in der Lage sind, Aufsichtsratsmandate kompetent und verantwortungsvoll wahrzunehmen. Deshalb

\section{Trotzdem müssen wir feststellen, dass aktive Gleich- stellungspolitik in Deutschland auf den öffentlichen Bereich begrenzt ist.}

übergebe ich im Verlauf des Abends eine Liste „Frauen in der Wirtschaft“ an Dr. Gerhard Cromme, den Vorsitzenden der Regierungskommission Deutscher Corporate Governance Kodex. Diese Liste enthält die Namen von mehr als 400 Frauen in Führungspositionen deutscher Unternehmen, die vom djb recherchiert wurden.

Unsere Erwartung ist, dass die Unternehmen ihre Politik bei der Besetzung von Aufsichtsräten deutlich verändern. Wir möchten dieses Anliegen ohne eine gesetzliche Quotenregelung umsetzen. Wir sind aber auch realistisch genug in unserer Einschätzung der Situation in Deutschland und in Betrachtung der Erfahrungen insbesondere in den skandinavischen Ländern, um eine Quote nicht auszuschließen!

Ich danke Ihnen. 